Frank Brückel

Pädagogische Hochschule Zürich

\title{
Regula Spirig
}

Pädagogische Hochschule Zürich

\section{Auf die Haltung kommt es an!}

\section{Zum Umgang mit Haltungen und Emotionen in der schulischen Führungsarbeit}

DOI: https://doi.org/10.53349/sv.2021.i2.a105

Eine der grossen Herausforderungen für schulische Führungskräfte ist der Umgang mit Haltungen und Emotionen. Der vorliegende Beitrag beschreibt, was unter Haltungen und Emotionen verstanden wird, wie sie zusammenhängen und wie ihnen als Führungsperson proaktiv begegnet werden kann. Je mehr eine Führungskraft um vorhandene Haltungen weiss, desto eher kann sie Emotionen proaktiv vorausahnen und entsprechende Strategien ergreifen.

Haltungen, Emotionen, Schulentwicklung, Schulführung

„When resistance appears, it is time to listen carefully to find out what the trouble is." Paul R. Lawrence, Harvard Business Review, 1969

Eine der grossen Herausforderungen für schulische Führungskräfte sowohl in der täglichen Führungsarbeit wie bei allen Fragen rund um die Aufgleisung neuer Schulentwicklungsprojekte ist der Umgang mit Haltungen und Emotionen. In den letzten Jahren haben sich die Autor*innen daher verstärkt der Bedeutung von Haltungen und Emotionen in Schulentwicklungsprozessen zugewandt und sich die Fragen gestellt, was darunter verstanden wird, wie sie zusammenhängen und wie ihnen proaktiv begegnet werden kann. Sie kommen zum Schluss, dass Haltungen der Boden sind, auf den jede Veränderung fällt. Sind sie einem Thema zugewandt, dann sind entsprechende Entwicklungen einfacher zu implementieren, da sie in der 


\section{\# schuleverantworten}

führungskultur_innovation_autonomie

Regel mit positiven Emotionen gekoppelt sind. Sind sie skeptisch oder ablehnend, wird die Umsetzung mit deutlich mehr Herausforderungen und Widerständen verbunden sein.

\section{Haltungen}

Der Begriff Haltung gehört zum täglichen Sprachgebrauch. In der Auseinandersetzung mit Literatur aus Soziologie und Pädagogik zeigt sich, dass unklar ist, was unter Haltungen zu verstehen ist, wie man sie erwirbt und wie und ob sie veränderbar sind (Kuhl et al., 2014). Fiegert und Solzbacher (2014, S. 25) bezeichnen Haltung als „,'Containerbegriff', der unverbindlich und beliebig mit wechselnden Inhalten gefüllt wird“. Dieser wird durch verschiedene Konzepte gespeist, es gibt keinen fachlichen Konsens darüber, was genau gemeint ist. Zudem zeigt es sich, dass Haltungen kaum von ,subjektiven Theorien', ,berufsbezogenen Überzeugungen' oder ,professionellen Haltungen' zu unterscheiden sind (vgl. Groeben \& Schlee, 1988; Schwarzer-Petruck, 2014; Reusser \& Pauli, 2014; Kuhl et al., 2014).

\section{Vier Dimensionen von Haltung}

In der Aufarbeitung und Verdichtung dieser Diskurse lassen sich vier verschiedene Dimensionen von Haltung unterscheiden. Diese geben den im Schulfeld handelnden Akteur*innen sowohl individuell als auch kollektiv bzw. professionell Orientierung und Handlungssicherheit (vgl. Reusser \& Pauli, 2014, S. 644 in Bezug auf Terhart, 2007).

- Persönliche Haltung: Unter persönlicher Haltung sind in Anlehnung an Reusser und Pauli (2014) und Schwer et al. (2014) biografisch geprägte Überzeugungen gemeint, die eine subjektive Selbst- und Weltsicht hervorbringen. Diese tragen massgeblich zur Stabilität des Handelns und zur Identität jedes Menschen bei.

- Professionsbezogene Haltung: Haltungen sind nicht nur individuell. Sie können auch professioneller Natur sein. Reusser und Pauli (2014, S. 645) schreiben dazu, dass Lehrpersonen viele Situationen in ihrem Beruf ähnlich wahrnehmen, Herausforderungen gleichartig lösen und in ,sozialer Abstimmung' handeln.

- Haltung des Teams: Unter Rückgriff auf Arbeiten zu Schulklima, Schulkultur und SchoolImprovement Capacity (z.B. Fend, 2008; Böhme et al., 2015; Maag Merki, 2017) kann konstatiert werden, dass es an jeder Schule eine Team-Haltung gibt. Darunter verstehen wir, in Anlehnung an Fend (2008, S. 201), die Mentalität eines Kollegiums, welche sich in der Ausprägung einer gemeinsamen pädagogischen Grundhaltung zeigt.

- Gesellschaftlicher Kontext: Es muss berücksichtigt werden, dass Schulen immer auch in einem gesellschaftlichen Kontext angesiedelt sind. Das heisst, dass es gesellschaftliche Aushandlungsprozesse darüber gibt, was Schule „darf und soll“ (Fend, 2006, S. 45ff). Dieser Kontext hat einen Einfluss sowohl auf die individuelle, auf die professionelle sowie auf die Haltung des Teams. 


\section{\# schuleverantworten}

\section{Erstes Zwischenfazit}

Haltungen sind geprägt durch individuelle biografisch entstandene Überzeugungen, die berufsbezogene Sozialisation, die jeweilige Schulkultur und gesellschaftliche Einflüsse. Was und wie genau Haltungen wirken, ist von Mensch zu Mensch, von Schule zu Schule und von Projekt zu Projekt unterschiedlich. Im Überblick können die vier Dimensionen von Haltungen wie folgt dargestellt werden:

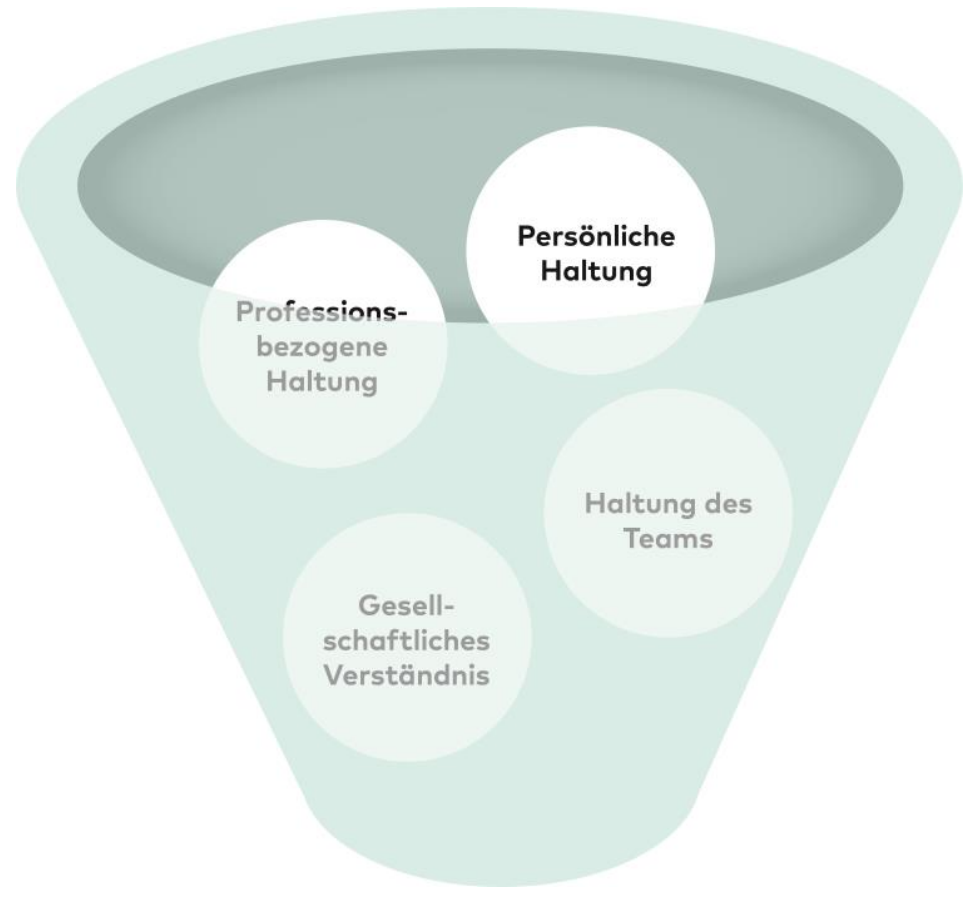

Abbildung 1: vier Dimensionen von Haltungen (eigene Darstellung)

\section{Emotionen}

Haltungen sind nicht statisch oder neutral. Reusser und Pauli (2014, S. 644) bezeichnen sie in Bezug auf andere Autor*innen als „emotional aufgeladene mentale Konfigurationen mit normativ-evaluativem Charakter". In der Konsequenz sind diese psychologische ,Frames', ,Filter' und ,Barrieren', "die sich in der Regel nur gegen Widerstände, Druck und Krisen verändern lassen“ (ebd.). Aus Sicht der Autor*innen gilt das nicht nur für negative Kräfte, auch positive Erfahrungen können Haltungen verändern. Sowieso reicht es nicht, Haltungen allein zu verstehen, da sie immer mit Emotionen verschränkt sind.

Im Folgenden wird zunächst beschrieben, was Emotionen sind, wie Haltungen und Emotionen (positive wie negative) zusammenhängen und Handlungen auslösen, um uns danach der Frage zuzuwenden, wie das Zusammenspiel von Haltungen und Emotionen möglichst proaktiv in der schulischen Führungsarbeit berücksichtigt werden kann. 


\section{\# schuleverantworten}

\section{Was sind Emotionen $?^{1}$}

Schwarzer-Petruck (2014) beschreibt Emotionen in Bezug auf Zimbardo et al. (2004) als ein komplexes Muster von körperlichen und mentalen Veränderungen, die eine Antwort auf bedeutsam wahrgenommene Situationen darstellen. Diese werden von Scherke (2009) in folgende fünf Dimensionen unterteilt:

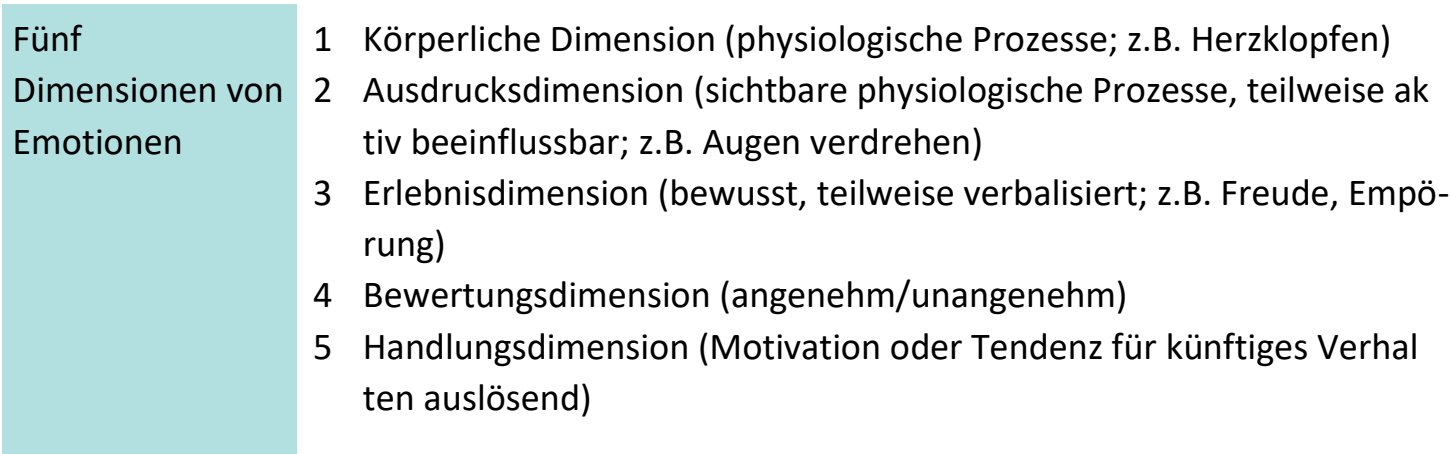

Demnach sind Emotionen nicht nur körperliche Signale, sondern können Handlungen auslösen und einen Prozess erheblich beeinflussen (Scherke, 2009). Was die Beschäftigung mit Emotionen ausserdem bedeutsam macht, ist, dass sie die Sichtweise auf eine Situation häufig dominieren (Schnabel, 2012a). Das heisst, dass nicht die kognitive Bewertung im Vordergrund steht, sondern die emotionale. Beispielsweise kann die Angst vor Versagen oder Bedeutungsverlust im Rahmen von Schulentwicklungsprojekten die fachlich-inhaltliche Diskussion entscheidend blockieren.

\section{Auslöser von Emotionen und daraus resultierende Handlungen}

Die Entstehung einer Handlung kann als Prozess beschrieben werden: Ein Ereignis wird durch den Filter der Haltungen eingeschätzt. Aufgrund der (Nicht-)Passung zwischen auslösendem Ereignis und Haltungen werden Emotionen ausgelöst, die dann Handlungen (mit-)steuern. Abbildung 2 zeigt den Prozess vom auslösenden Moment zur Handlung im Überblick auf, der danach beschrieben wird. 


\section{\# schuleverantworten}

führungskultur_innovation_autonomie
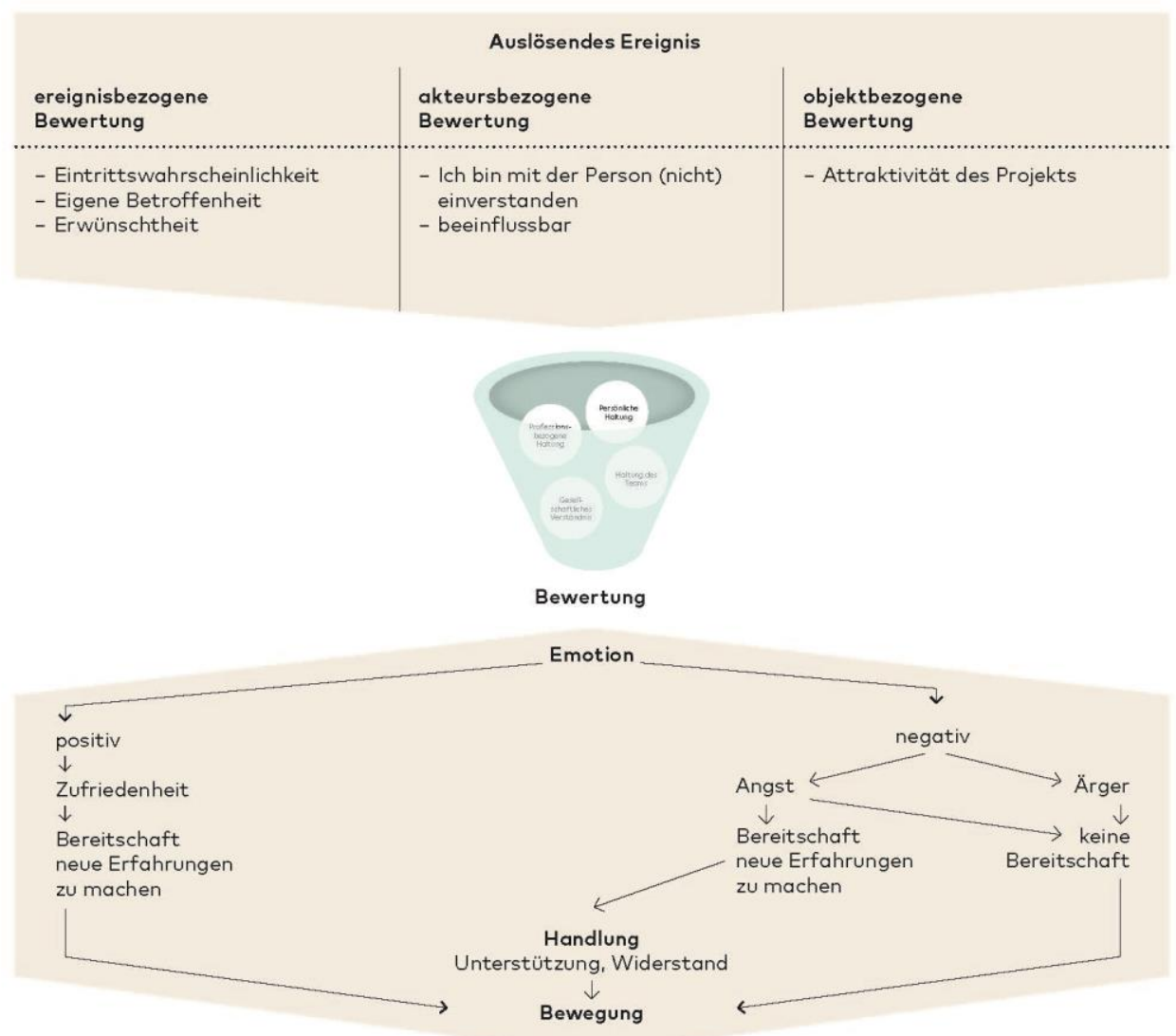

Abbildung 2: Vom auslösenden Moment zur Handlung (eigene Darstellung)

Nach Scheve (2012, S. 122) werden Emotionen nicht direkt durch Ereignisse, sondern durch deren Bewertungen ausgelöst. Ob diese eher positiv, ambivalent oder eher negativ ausfallen, hängt mit den oben beschriebenen Haltungen zusammen.

Verschiedene Autor*innen unterscheiden zwischen drei emotionsauslösenden Kategorien von Bewertungen:

Ereignisbezogene Auslöser
Ereignisbezogene Emotionen gründen auf den Einschätzungen, inwieweit Personen vom Ereignis betroffen sind, mit welcher Wahrscheinlichkeit es eintreffen wird und wie hoch die Erwünschtheit ist (zufrieden/unzufrieden) (vgl. Scheve 2012, S. 122). 
Akteursbezogene Auslöser

Objektbezogene Auslöser
Akteursbezogene Emotionen hängen davon ab, „wer oder was für ein bestimmtes Ereignis verantwortlich gemacht wird" und ob dieses Ereignis als beeinflussbar erscheint (befürworten/ablehnen) (vgl. Schnabel, 2012b, S. 21; Scheve, 2012, S. 122). Objektbezogene Emotionen beziehen sich nach Scheve (2012, S. 122) auf Objekte bzw. Themen oder Projekte, die als attraktiv oder unattraktiv eingeschätzt werden (mögen/nicht mögen).

Als Führungsperson kann man Einfluss auf diese auslösenden Momente nehmen, wenn man sich zum Beispiel mit folgenden Fragen auseinandersetzt:

- Welche Kolleg*innen sind von einem neuen Projekt betroffen?

- Wer führt das Projekt ein und wer gestaltet mit?

- Wie anschlussfähig ist das Projekt und welchen Mehrwert bringt es der Schule?

Wie bereits ausgeführt, sind die für die Bewertung verantwortlichen Haltungen nicht nur individuell, sondern auch kollektiv. Deshalb ist es möglich, dass eine Gruppe (z.B. ein Team) bestimmte Ereignisse vergleichbar bewertet und auf eine ähnliche Weise affektiv beziehungsweise emotional reagiert (vgl. Scheve 2012, S. 125). Solche emotionalen Reaktionen können sowohl unmittelbar und automatisch (assoziativ) als auch mit Verzögerung und ressourcenintensiv (deliberativ) vorkommen (vgl. Scheve 2012; Schwarzer-Petruck 2014).

\section{Wirkung von Emotionen auf Handlungen}

Nachdem aufgezeigt wurde, wie Emotionen entstehen, soll im Folgenden beschrieben werden, wie sie positiv bzw. negativ wirken.

Wird ein auslösendes Ereignis als übereinstimmend mit den eigenen Haltungen und sinnstiftend bewertet, entstehen eher positive Emotionen (vgl. Schwarzer-Petruck, 2014, S. 67; Straßheim, 2012, S. 57). In Anlehnung an andere Forschungsarbeiten zeigt Schwarzer-Petruck (2014, S. 84ff.) auf, dass solche das Repertoire des Denkens und Handelns in mehrfacher Hinsicht erweitern: Sie erhöhen die Bereitschaft, eigene Grenzen zu überschreiten und neue Erfahrungen zu machen, sind Auslöser für die Integration neuer Informationen und Ansichten sowie das Anvisieren neuer Ziele. Sie lösen Bewegung aus (vgl. Abb. 2).

Im Gegensatz dazu führen negative Emotionen zu einer Einschränkung des „momentanen Gedanken-Handlungsrepertoires einer Person“" (Schwarzer-Petruck, 2014, S. 84). Im Wesentlichen kann zwischen Angst und Ärger unterschieden werden. Angst entsteht, wenn die Kontrollierbarkeit und die eigenen Ressourcen als ungenügend empfunden werden und dadurch die Emotion der Machtlosigkeit entsteht. Wird diese Machtlosigkeit auf die eigene Person zurückgeführt, entstehen Gefühle der Scham, Unsicherheit, Hilflosigkeit. Gleichzeitig beschreiben sowohl Scherke (2009), Schützeichel (2012) wie auch Schwarzer-Petruck (2014), dass Angst nicht ausschliesslich negative Konsequenzen haben muss, insbesondere dann, wenn sie 


\section{\# schuleverantworten}

führungskultur_innovation_autonomie

zu Aktionen führt, die zur „Eindämmung der Furcht“ dienen (Scherke, 2009). Im Idealfall führt die Überwindung zu mehr Selbstvertrauen und verringert die Emotion der Machtlosigkeit.

Werden andere für die Machtlosigkeit verantwortlich gemacht, kann es zu Ärger und Feindseligkeit bis hin zu Widerstand kommen (Scherke, 2009; Dehne, 2012, S. 139). Scheve (2012, S. 117) zeigt auf, dass Wut und Ärger in den meisten Fällen dadurch entstehen, dass angenommene soziale Normen und Konventionen verletzt werden. Im Unterschied zur Angst führt nach Schützeichel (2012, S. 238ff.) der Ärger dazu, unreflektiert an der eigenen Haltung festzuhalten, also nicht zu einer Bereitschaft, über mögliche Handlungsalternativen nachzudenken oder diese umzusetzen.

\section{Zweites Zwischenfazit}

Haltungen sind der Boden, auf den jede Veränderung fällt, Emotionen sind positive oder negative Treiber. Dabei ist es wichtig, genauer zu fassen, was unter Haltungen und Emotionen zu verstehen ist und wie beide zusammenhängen.

Unterschieden wird zwischen vier Dimensionen von individuellen und kollektiven Haltungen, diese bilden einen soliden und dauerhaften Rahmen, der Orientierung und Handlungssicherheit gibt (vgl. Reusser \& Pauli, 2014 in Bezug auf Terhart, 2007).

Ereignisse (z.B. die Ankündigung eines neuen Projekts) werden immer aufgrund des Filters der Haltungen eingeschätzt und bewertet. Je nach Übereinstimmung führen sie zu positiven, ambivalenten oder negativen Emotionen. Bewertungen geschehen aufgrund von drei emotionsauslösenden Kategorien: ereignisbezogene (Wahrscheinlichkeit und Erwünschtheit), akteurs- oder personenbezogene (Verantwortlichkeit und Ressourcen), objektbezogene Auslöser (Attraktivität). Die Bewertung erfolgt teilweise unmittelbar und automatisch, teilweise mittelbar und mit Verzögerung. Positive Emotionen unterstützen die Bereitschaft zur Erweiterung des Handlungsrepertoires, negative wirken einschränkend.

\section{Umgang mit Haltungen und Emotionen in der Schulentwick- lungsarbeit}

Wie also kann proaktiv mit Haltungen und Emotionen verfahren werden? Die bisherigen Ausführungen und beigezogenen Publikationen geben eine Reihe von Hinweisen, die bei der Führung von Veränderungsprozessen berücksichtigt werden können. Im Kern geht es darum, die vier Dimensionen von Haltung besser einschätzen zu können, erwartbare Emotionen daraus abzuleiten und entsprechende Handlungsstrategien zu entwickeln.

\section{Durch den Dialog Haltungen explizit machen}

Verschiedene Autor*innen (Schwarzer-Petruck, 2014; Reusser \& Pauli 2014; Nonaka et al., 2012; Scharmer, 2020) weisen darauf hin, dass die Bewusstmachung individueller und kollek- 
tiver Haltungen eine sehr wichtige Arbeit ist. Dafür braucht es genügend Gelegenheit zum gegenseitigen Austausch über Sinn (oder Unsinn) des kommenden Projekts. Denn erst im Austausch mit anderen und durch die Versprachlichung werden Haltungen explizit gemacht. Allerdings - und das ist sehr wichtig - muss es um einen Dialog gehen (Bohm et al., 2011). Kennzeichnend für einen Dialog (im Gegensatz zu einer Diskussion) ist, so schreibt Bohm (2011, S. 27ff.), dass die Gesprächsteilnehmer*innen in der Lage sein sollen, einander uneingeschränkt und vorurteilsfrei zuzuhören, ohne zu versuchen, sich gegenseitig zu beeinflussen. Es geht nicht um ein ,besser oder schlechter', sondern um eine Explizitmachung eigener Ansichten, innerer Bilder und die Offenlegung von Haltungen, also dem Verstehen des Gegenübers.

Die Brücke von explizit gemachten Haltungen zum Führungshandeln geschieht durch die Beobachtung und Analyse. Wichtig ist zu erkennen, welche Emotionen mitspielen und wodurch sie ausgelöst werden. Sind die Kolleg*innen zum Beispiel darüber im Dialog, ob und wie sie vom neuen Projekt betroffen sind (ereignisbezogen)? Oder beschäftigen sie sich lediglich damit, wie die Führungsperson das neue Projekt einzuführen versucht (akteursbezogen)? Erachten die Kolleg*innen das neue Projekt als anschlussfähig an ihre pädagogische Arbeit und sehen sie einen Mehrwert (objektbezogen)?

Ergibt die Beobachtung, dass eine positive Bewertung des Projekts stattfindet, können die nächsten Schritte angegangen werden. Ist die Bewertung skeptisch oder sogar negativ, lohnt sich eine genauere Analyse des weiteren Vorgehens. Sind Ängste im Spiel, gilt es, diese aufzufangen und Möglichkeiten zu schaffen, sie zu überwinden. Bei Ärger hingegen ist genau zu prüfen, woher dieser kommt, weil ansonsten projektbezogene Handlungen nur schwer möglich sind.

\section{Neue Erfahrungen ermöglichen und Daten nutzen}

Reusser und Pauli (2014, S. 646) schreiben, dass neue Informationen „weiterhin vor dem Hintergrund der ,alten', langjährig bewährten Überzeugungen assimiliert" (gefiltert) werden, was sie als eine der Ursachen für die unzureichende Umsetzung von Reformen sehen. Um solche ,alten' Haltungen in Bewegung zu bringen, können gezielte Interventionen Dialoge beflügeln, etwa, indem neue Erfahrungen ermöglicht werden, z.B. eigene Erfahrungen (ausprobieren), Erfahrungen von Dritten (beobachten). Dieses ,Erfahren lassen' hat im Zusammenhang mit Haltungen und Emotionen eine wichtige Funktion. Verkürzt ausgedrückt können sich Menschen einen neuen Alltag manchmal nicht vorstellen, wenn sie keine entsprechenden Erfahrungen dazu gemacht haben.

Und schliesslich können Dialoge durch den Beizug von Daten angereichert werden (vgl. z.B. Schildkamp, 2019). Nicht nur wissenschaftliche Ergebnisse, sondern auch eigene Befragungen oder Dokumentationen sind geeignet, um das Projekt auch datenbasiert und nicht ausschliesslich emotional zu bewerten. 
Führungspersonen können die Kombination von Dialog, Erfahrung und Daten so gestalten, dass ein gemeinsames Verständnis und eine gemeinsame Sprache im Team entsteht. Damit unterstützen sie die Erweiterung des Handlungsrepertoires und schaffen die Grundvoraussetzung für Veränderungen. Es geht ausdrücklich nicht darum, dass alle Kolleg*innen eines Teams immer gleich agieren, sondern, dass sie ihre Handlungen an einem gemeinsamen $\mathrm{O}-$ rientierungsrahmen ausrichten. Werden die Ziele des Projekts im Ergebnis als sinnvoll erachtet, steht den nächsten Schritten nichts im Weg. Ist das Gegenteil der Fall, sollten die Projektziele noch einmal kritisch geprüft werden.

\section{Kontexte berücksichtigen}

Schulen sind gesellschaftliche Institutionen, in ein Mehrebenensystem eingebunden und gelten als Expertenorganisationen. Dies hat einen erheblichen Einfluss darauf, welche Haltungen und Emotionen erwartbar sind. Da es sowohl zur Einbindung von Schulen im Mehrebenensystem wie auch zur Wirkweise von Expertenorganisationen umfangreiche Publikationen gibt, sollen hier nur die wesentlichen Eckpunkte in Bezug auf mögliche (positive oder negative) Haltungen und Emotionen skizziert werden.

In föderalen Systemen ist das Bildungswesen als „ausgeprägtes Mehrebenensystem“ (Appius \& Nägeli, 2017, S. 35ff.) konzipiert, wobei jede Ebene ihre ganz spezifische Funktion hat und ein Zusammenspiel der Ebenen unabdingbar ist. Die Herausforderung bei diesem Zusammenspiel besteht darin, dass jede dieser Ebenen ihrer eigenen inneren Logik folgt und ihre je eigene spezifische Perspektive auf das Feld Schule hat (Bonsen, 2016, S. 303). Diese unterschiedlichen Sichtweisen und Logiken stellen für Schulentwicklungsvorhaben häufig eine sehr grosse Herausforderung dar, da sie bei Veränderungsprozessen immer wieder zu Missverständnissen bis hin zu handfesten Interessenskonflikten führen können (Langer, 2008, S. 8). Die Herausforderung lässt sich abschwächen, wenn die verantwortliche Projektleitung sowohl die Funktionsweise des Bildungswesens wie auch die jeweiligen Interessen kennt und so weit als möglich zusammenführen kann.

Darüber hinaus ist es wichtig, zu berücksichtigen, dass Einzelschulen Merkmale von Expertenorganisationen aufweisen. Expert*innen haben zum Beispiel eine hohe Autonomie in der Ausübung ihrer Tätigkeit, daher ist ihre Motivation von entscheidender Bedeutung. Diese entspringt vor allen Dingen aus ihrer Kerntätigkeit (dem Fach, der Arbeit mit der Klasse, etc.) und weniger aus der Zugehörigkeit zu einer Organisation. Wichtig ist zudem zu berücksichtigen, dass Macht durch Expertise in der Regel höher eingeschätzt wird als Macht durch hierarchischen Rang (Rybnicek et al., 2016, S. 229 in Bezug auf Anand et al., 2013). Vereinfacht kann gesagt werden, dass in Expertenorganisationen die Macht der Argumentation deutlich höher zu gewichten ist als das Argument der Macht.

\section{Drittes Fazit}

Um in einem Schulentwicklungsprozess mit Haltungen und Emotionen proaktiv verfahren zu können, ist es zunächst wichtig, möglichst viel zu vorhandenen Haltungen und den damit ver- 
bundenen Emotionen zu erfahren und diese den Zielen des kommenden Projekts zuzuordnen. Durch einen Dialog über den Sinn des kommenden Projekts werden die Teammitglieder aufgefordert, ihre Vorstellungen explizit zu machen. Eine positive Einschätzung wird die Bereitschaft des Teams fördern, sich auf neue Praktiken einzulassen. Ängste können durch das Aufzeigen von Handlungsoptionen abgefedert werden, wohingegen Ärger eher zu einer Verweigerung führt und es oft einer genauen Analyse dessen bedarf, was ihn auslöst. Zusätzlich zu den Dialogen können die Ermöglichung neuer Erfahrungen und die Bereitstellung von Daten helfen, Haltungen kritisch konstruktiv zu hinterfragen und Handlungsoptionen aufzuzeigen.

Je besser der Kontext bekannt ist, in dem die Veränderung umgesetzt werden soll, desto eher können negative Reaktionen verhindert werden: Schulen als Expertenorganisationen sind eingebunden in ein Mehrebenensystem. Beide weisen spezifische Merkmale auf, die Auswirkungen auf Haltungen und Emotionen haben und deren Berücksichtigung die Führung von Veränderungsprozessen erheblich erleichtern kann.

\section{Unberechenbarkeit der Praxis}

Wir vertreten in dem vorliegenden Beitrag die These, dass Haltungen der Boden sind, auf den jede Veränderung fällt und damit zusammenhängende Emotionen positive oder negative Treiber darstellen. Daher - so unsere Argumentation - ist es wichtig, sich damit auseinanderzusetzen, was unter Haltungen und Emotionen zu verstehen ist, woher sie kommen, wie man sie erkennt und wie man ihnen begegnen kann. Denn je mehr eine Führungsperson um vorhandene Haltungen weiss, desto eher kann sie Emotionen vorausahnen und entsprechende Strategien proaktiv ergreifen.

Sollte hierbei der Eindruck entstehen, dass Haltungen in jedem Falle erkannt und Emotionen daher in den meisten Fällen in positive Bahnen gelenkt werden können, wollen wir in Anlehnung an Altrichter und Maag Merki (2016, S. 3) vor "naivem Planungsoptimismus" warnen. Zwar sind Haltungen und Emotionen bis zu einem gewissen Grad erklär- und vorhersehbar. Sie beinhalten jedoch immer auch Komponenten, die Unberechenbarkeit zum Normalfall werden lassen (vgl. Reckwitz, 2003). Jede Situation ist anders, man kann Menschen sehr gut kennen, aber nie endgültig entschlüsseln. Auch die grösste Routine wird nicht dazu führen, dass genau vorausgesagt werden kann, wie Menschen in Situationen handeln und mit welchen Emotionen sie reagieren. Dennoch gibt die Auseinandersetzung mit Haltungen und Emotionen eine beschränkte Sicherheit, die Reaktionen erwartbarer machen.

\section{Literaturverzeichnis}

Altrichter, H. \& Maag Merki, K. (2016). Steuerung der Entwicklung des Schulwesens. In H. Altrichter \& K. Maag Merki (Hrsg.), Handbuch: Band 7. Handbuch Neue Steuerung im Schulsystem (2. Aufl., S. 127). Springer VS. 


\section{\# schuleverantworten}

führungskultur_innovation_autonomie

Appius, S. \& Nägeli, A. (2017). Schulreformen im Mehrebenensystem: Eine mehrdimensionale Analyse von Bildungspolitik. Educational Governance: v.35. Springer Fachmedien Wiesbaden. https://ebookcentral.proquest.com/lib/gbv/detail.action?doclD=4790536

Bohm, D., Nichol, L. \& Grube, A. (Hrsg.). (2011). Der Dialog: Das offene Gespräch am Ende der Diskussionen (6. Aufl.). Klett-Cotta. http://swbplus.bsz-bw.de/bsz26186369xcov.htm

Böhme, J., Hummrich, M. \& Kramer, R.-T. (2015). Schulkultur. Springer Fachmedien Wiesbaden. https://doi.org/10.1007/978-3-658-03537-2

Bonsen, M. (2016). Schulleitung und Führung in der Schule. In H. Altrichter \& K. Maag Merki (Hrsg.), Handbuch: Band 7. Handbuch Neue Steuerung im Schulsystem (2. Aufl., S. 301-323). Springer VS.

Dehne, M. (2012). Wieso, weshalb, warum? In A. Schnabel \& R. Schützeichel (Hrsg.), Emotionen, Sozialstruktur und Moderne (S. 139-158). VS Verlag für Sozialwissenschaften. https://doi.org/10.1007/978-3-531-93443-3_7

Fend, H. (2006). Neue Theorie der Schule: Einführung in das Verstehen von Bildungssystemen. Lehrbuch. VS Verlag für Sozialwissenschaften.

Fend, H. (2008). Schule gestalten: Systemsteuerung, Schulentwicklung und Unterrichtsqualität. Lehrbuch. VS Verlag für Sozialwissenschaften. https://doi.org/10.1007/978-3-531-90867-0

Fiegert, M. \& Solzbacher, C. (2014). „Bescheidenheit und Festigkeit des Charakters ...“ Das Konstrukt Lehrerhaltung aus historischsystematischer Perspektive. In C. Schwer \& C. Solzbacher (Hrsg.), Professionelle pädagogische Haltung: Historische, theoretische und empirische Zugänge zu einem viel strapazierten Begriff (S. 17-45). Julius Klinkhardt.

Groeben, N. \& Schlee, J. (1988). Das Forschungsprogramm Subjektive Theorien: Eine Einführung in die Psychologie des reflexiven Subjekts. Francke.

Kuhl J., Schwer, C. \& Solzbacher, C. (2014). Professionelle pädagogische Haltung: Persönlichkeitspsychologische Grundlagen. In C. Schwer \& C. Solzbacher (Hrsg.), Professionelle pädagogische Haltung: Historische, theoretische und empirische Zugänge zu einem viel strapazierten Begriff (S. 79-106). Julius Klinkhardt.

Langer, R. (Hrsg.). (2008). Educational Governance: Bd. 6. "Warum tun die das?": Governanceanalysen zum Steuerungshandeln in der Schulentwicklung. VS Verlag für Sozialwissenschaften.

Maag Merki, K. (2017). School Improvement Capacity als ein Forschungsfeld der Schulentwicklungsund Schuleffektivitätsforschung: Theoretische und methodische Herausforderungen. In U. Steffens, H. Fend \& K. Maag Merki (Hrsg.), Beiträge zur Schulentwicklung. Schulgestaltung: Aktuelle Befunde und Perspektiven der Schulqualitäts- und Schulentwicklungsforschung: Grundlagen der Qualität von Schule 2 (S. 269-286). Waxmann.

Nonaka, I., Takeuchi, H. \& Mader, F. (2012). Die Organisation des Wissens: Wie japanische Unternehmen eine brachliegende Ressource nutzbar machen (2. Aufl.). Management. Campus.

Reckwitz, A. (2003). Grundelemente einer Theorie sozialer Praktiken: Eine sozialtheoretische Perspektive. Zeitschrift für Soziologie, 32(4), 282-301.

Reusser, K. \& Pauli, C. (2014). Berufsbezogene Überzeugungen von Lehrerinnen und Lehrern. In E. Terhart, H. Bennewitz \& M. Rothland (Hrsg.), Handbuch der Forschung zum Lehrerberuf (2. überarb. u. erw. Aufl., S. 642-661). Waxmann.

Rybnicek, R., Bergner, S. \& Suk, K. (2016). Führung in Expertenorganisationen. In J. Felfe \& R. van Dick (Hrsg.), Handbuch Mitarbeiterführung (S. 227-237). Springer Berlin Heidelberg. https://doi.org/10.10- 


\section{\# schuleverantworten}

führungskultur_innovation_autonomie

07/978-3-642-55080-5_42

Scharmer, C. O. (2020). Theorie U - Von der Zukunft her führen: Presencing als soziale Technik (5. Aufl.). Management. Carl Auer.

Scherke, K. (2009). Emotionen als Forschungsgegenstand in der deutschsprachigen Soziologie. VS Verlag für Sozialwissenschaften.

Scheve, C. von. (2012). Die sozialen Grundlagen der Emotionsentstehung: Kognitive Strukturen und Prozesse. In A. Schnabel \& R. Schützeichel (Hrsg.), Emotionen, Sozialstruktur und Moderne (S. 115137). VS Verlag für Sozialwissenschaften. https://doi.org/10.1007/978-3-531-93443-3_6

Schildkamp, K. (2019). Data-based decision-making for school improvement: Research insights and gaps. Educational Research, 61(3), 257-273. https://doi.org/10.1080/00131881.2019.1625716

Schnabel, A. \& Schützeichel, R. (Hrsg.). (2012a). Emotionen, Sozialstruktur und Moderne. Springer VS.

Schnabel, A. (2012b). Emotionen, Sozialstruktur und Moderne - ein spannungsvolles Wechselverhältnis.: Zur Einleitung. In A. Schnabel \& R. Schützeichel (Hrsg.), Emotionen, Sozialstruktur und Moderne (S. 9-27). VS Verlag für Sozialwissenschaften.

Schützeichel, R. (2012). Emotionen in Handlungen. In A. Schnabel \& R. Schützeichel (Hrsg.), Emotionen, Sozialstruktur und Moderne (S. 227-255). VS Verlag für Sozialwissenschaften. https://doi.org/10.1007/978-3-531-93443-3_11

Schwarzer-Petruck, M. (2014). Emotionen und pädagogische Professionalität: Zur Bedeutung von Emotionen in Conceptual-Change-Prozessen in der Lehrerbildung. Zugl.: Jena, Univ., Diss., 2012. Springer VS.

Schwer, C., Solzbacher, C. \& Behrensen Birgit. (2014). Annäherungen an das Konzept „Professionelle pädagogische Haltung": Ausgewählte theoretische und empirische Zugänge. In C. Schwer \& C. Solzbacher (Hrsg.), Professionelle pädagogische Haltung: Historische, theoretische und empirische Zugänge zu einem viel strapazierten Begriff (S. 47-77). Julius Klinkhardt.

Straßheim, J. (2012). Emotionen nach Alfred Schütz. In A. Schnabel \& R. Schützeichel (Hrsg.), Emotionen, Sozialstruktur und Moderne (S. 49-73). VS Verlag für Sozialwissenschaften. https://doi.org/10.1007/978-3-531-93443-3_3

Zimbardo, P. G., Gerrig, R. J. \& Graf, R. (Hrsg.). (2004). Pearson-Studium ps, Psychologie. Psychologie (16. Aufl.). Pearson-Studium.

\section{Anmerkungen}

${ }^{1}$ An dieser Stelle wird nicht auf psychologische Arbeiten zum Thema Emotionen eingegangen, sondern ausschliesslich soziologische und pädagogische Literatur hinzugezogen.

\section{Autor*innen}

Frank Brückel, Prof. Dr.

arbeitet an der Pädagogischen Hochschule Zürich im Arbeitsschwerpunkt Schulentwicklung. Dabei unterstützt er Gemeinden und Schulen bei den unterschiedlichsten Veränderungsprozessen. Sein besonderes Interesse liegt dabei im Wissenstransfer von Forschungswissen in 
die tägliche Schulentwicklungsarbeit.

Kontakt: frank.brueckel@phzh.ch

Regula Spirig Esseiva, lic. phil., arbeitet an der Pädagogischen Hochschule Zürich im Arbeitsschwerpunkt Schulentwicklung. Sie begleitet Schulen und Teams bei Entwicklungsprozessen in den Themen Tagesschule und kompetenzorientierte Beurteilung. Daneben ist sie als Mentorin in der berufspraktischen Ausbildung.

Kontakt: regula.spirig@phzh.ch 